\title{
Resumen
}

La presente investigación se propone, analizar la variación del valor de las acciones pertenecientes a empresas del sector bancario chileno, que cotizan en bolsa, producto de la oferta pública de acciones, desde el año 2004. Describe las características de las ofertas públicas, de acuerdo a la información entregada por la Superintendencia de Valores y Seguros, realizadas a partir del año 2004, en el sector bancario chileno. Correlaciona el valor de cierre histórico de las acciones del sector bancario, durante el período en que se genera la OPA (el período antes y después) y explica, mediante la evidencia obtenida, las variaciones experimentadas en las acciones cotizadas en el mercado bursátil, que se han generado producto del lanzamiento de una OPA. Se concluye que la hipótesis planteada y el objeto de investigación ponen de manifiesto que existe una variación anormal en el mercado bursátil, ante la realización de una OPA, pero esta variación anormal, no es significativa para el rango de variación dado por la investigación (5\%).

Palabras claves: Variación de Acciones, Sector Bancario, Oferta Pública de Acciones, Correlación, Mercado Bursátil.

\section{EL VALOR DE LAS ACCIONES PRODUCTO DE UNA OPA EN EL SECTOR FINANCIERO}

\section{THE VALUE OF SHARES AS A RESULT OF AN IPO IN THE FI- NANCIAL SECTOR}

\author{
Elizabeth Belmar Del Canto ${ }^{1}$
}

\section{Abstract}

This investigation tries to analyze the variation in the value of shares belonging to companies of the Chilean banking sector, which quote in the stock market because of public offering of its shares since 2004. It describes the characteristics of public offers according to information given by the Superintendence of Securities and Insurance carried out from the year 2004 on, in the Chilean banking sector. It correlates the closing value of the historical shares during the period when the IPO generates (the period before and after). With the obtained evidence, it explains the variations experimented by the quoted shares in the stock market as a result of the launching of an IPO. Thus the hypothesis attempted and the objective of this investigation show that there is an abnormal fluctuation in the stock market when an IPO occurs, but this fluctuation is not significant for the range of variation given by this investigation. (5\%)

Key Words: Share fluctuation, Banking Sector, IPO (initial public offering), Correlation, Stock market.

${ }^{1}$ Alumna Tesista de la Carrera de Auditoría de la Universidad de Valparaíso, teniendo como profesor guía al Prof. Dra. Karime Chahuán Jiménez . 


\section{INTRODUCCIÓN.}

El 20 de diciembre del año 2000, se promulgó en el Diario Oficial la ley 19.705, que se encarga de regular la oferta pública de adquisición de acciones, también conocida como ley de OPA. Entre otras materias, implemento cambios a la Ley General de Bancos. De acuerdo a éstas, la Superintendencia de Bancos e Instituciones Financieras, adquieren la facultad de autorizar fusiones y tomas de control bancarias, que resulten en una "participación significativa de mercado", la cual es definida por esta entidad, como superior a $20 \%$. En caso de denegar una autorización, se requerirá un previo informe del Banco. También se podrá condicionar una autorización al cumplimiento de algunas exigencias referidas a la solvencia de las instituciones involucradas.

Según lo que se puede observar en los avisos de oferta pública, emitidos por la Superintendencia de Valores y Seguros, Ios bancos utilizan la oferta pública de adquisición de acciones para obtener una participación accionarial significativa, que les dé derecho a voto y toma de control de otras entidades bancarias 0 para adquirirlas en su totalidad. Según Muñiz (2010) la sociedad que realiza la oferta, habitualmente, ofrece por las acciones un precio mayor que el del mercado, lo que puede producir una variación desconocida en la cotización de la compañía afectada por la OPA.

Por lo tanto, el propósito de esta investigación es analizar las variaciones que se han generado en el valor de las acciones, producto de la oferta pública de adquisición de acciones (OPA), en el sector bancario chileno. 


\section{HIPÓTESIS}

La publicación de Oferta Pública de Adquisición de Acciones (OPA), produce efectos significativos en la variación del valor de las acciones de las empresas objetivo.

\section{MERCADO DE VALORES}

EI Mercado Valores, según la SVS (2011) es representado, comúnmente como el lugar físico en el que se demandan, ofrecen y se intercambia algún tipo de productos. En cambio, la economía de mercado en Chile, da a conocer una definición mucho más amplia y lo representa como un conjunto de mecanismos, por medio del cual, los compradores y vendedores de un bien y/o servicio, están en contacto para comercializarlo.

El mercado de capitales según la SVS (2011) es donde se reúnen la oferta y la demanda para intercambiar los más conforman una infraestructura, de tal manera que permite a los oferentes de los recursos vender éstos a los demandantes.

El mercado de capitales chileno se distribuye en tres sectores, los cuales se dividen, según el regulador a cargo de su supervisión.

AFP: Administradoras de fondos de pensiones.

SAFP: Superintendencia de

Administradoras de Fondos de

Pensiones.

SBIF: Superintendencia de Bancos e Instituciones Financieras.

SVS: Superintendencia de Valores y

Seguros.

\begin{tabular}{|l|l|l|l|}
\hline \multicolumn{4}{|c|}{ Cuadro No1 Sectores del mercado } \\
\hline Sector & Oferta & Demanda & Regulador \\
\hline AFP & Compra deuda & & SAFP \\
\hline Bancario & $\begin{array}{l}\text { Créditos } \\
\text { (colocaciones) }\end{array}$ & $\begin{array}{l}\text { Depositan } \\
\text { tes(captación) }\end{array}$ & SBIF \\
\hline $\begin{array}{l}\text { Mercado de } \\
\text { Valores y seguros }\end{array}$ & $\begin{array}{l}\text { Valores de oferta } \\
\text { pública }\end{array}$ & & SVS \\
\hline
\end{tabular}

Fuente: Elaboración propia en base a SVS (2011)

diversos capitales y en las más diversas formas como el dinero, valores 0 activos financieros, y no solo interviene la oferta y la demanda, también existen intermediarios especializados, que a su vez son regulados y controlados. Por tanto, se puede decir que el mercado es el conjunto de regulaciones, instituciones, prácticas y personas que

\section{ESTRUCTURA DEL MERCADO}

A partir de la SVS (2011) el mercado está compuesto por la oferta y la demanda $y$, también existen intermediarios especializados, que a su vez son regulados y controlados por otros entes. En definitiva se puede decir que el mercado es el conjunto de 
regulaciones, instituciones, prácticas y reguladores, que conforman una estructura como la presentada a continuación: bonos y pagarés con diferentes denominaciones; la Tesorería General de la República, principalmente a través de bonos, el Instituto de Normalización

Figura $\mathrm{N}^{\circ} 1$ Estructura del Mercado

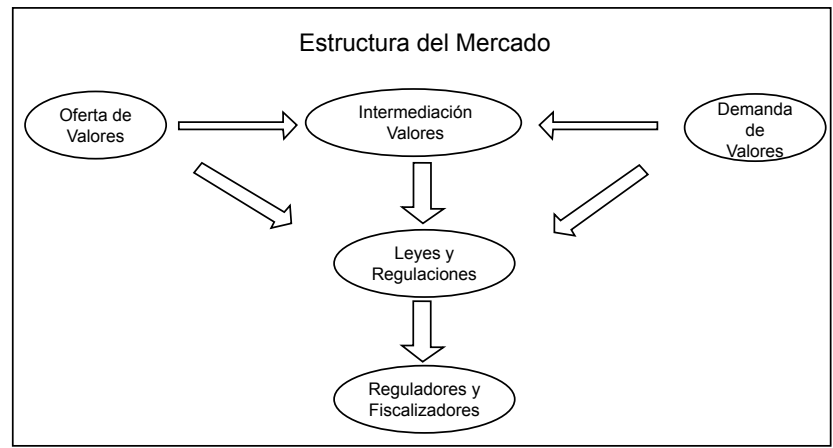

Fuente : Propia en base a información entregada por la SVS (2011)

\section{Emisores de valores}

En el Mercado de Valores se puede identificar claramente a los emisores que participan en él:

Sociedades Anónimas: Emiten títulos de acciones y títulos de renta fija, principalmente bonos y efectos de comercio. (SVS, 2011)

Bancos e Instituciones Financieras: participan en el mercado a través de la emisión de depósitos, letras hipotecarias y bonos bancarios, entre otros. (SVS, 2011)

Fondos Patrimoniales o Institucionales: Los fondos institucionales o patrimoniales constituidos en Chile y regulados por la SVS son: fondos mutuos, fondos de inversión, fondos de inversión de capital extranjero y fondos para la vivienda. (SVS, 2011)

Instituciones Públicas: las instituciones autorizadas para emitir valores de deuda son: el Banco Central de Chile, principalmente a través de
Previsional, que emite bonos de reconocimiento y las empresas públicas. (SVS, 2011)

Sociedades Securitizadoras: Son sociedades especiales, sujetas a la fiscalización de la SVS, que adquieren activos 0 derechos sobre flujos futuros con los cuales constituyen un patrimonio separado, que respalda la emisión de un bono securitizado, que se ofrece públicamente en el mercado. (SVS, 2011)

Emisores Extranjeros: Los valores extranjeros que se pueden inscribir y ofrecer, públicamente en Chile, son cuotas de fondos, acciones y Certificados de Depósitos de Valores (CDV). (SVS, 2011)

Intermediarios Corredores de bolsa: Son aquellos que operan dentro de la bolsa. Para ser miembros de una bolsa, los corredores de valores deben cumplir con las exigencias legales, con la normativa bursátil y adquirir una acción del centro bursátil en que desean 
operar. (SVS, 2011)

Agentes de valores: Son aquellos que operan fuera de la bolsa. (SVS, 2011)

Bancos y Sociedades Financieras: Son intermediarios y se deben regir por la Ley de Bancos, pero cuando se trata de una compra y venta de acciones, deben ser realizadas por corredores de Bolsa. No se deben inscribir en el registro de corredores, pero se deben regir por las leyes del mercado. (SVS, 2011)

\section{Instrumentos del Mercado}

Los instrumentos de mercado son aquellos que permiten obtener diversos tipos de crédito y están disponibles en la economía. Los instrumentos financieros, generalmente se transan en la Bolsa de Valores y son denominados "valores" o "títulos mobiliarios". Estos representan una parte del capital de la empresa 0 una deuda. (Bolsa de Santiago, 2011) .

Existen distintas clasificaciones de los instrumentos de mercado, pero, generalmente, se clasifican en dos:

\section{Renta Fija:}

Los instrumentos de renta fija son emitidos por entidades privadas 0 públicas con el objetivo de obtener recursos, la renta fija son títulos que representan obligaciones, donde se adquiere un compromiso de devolución de capital y, además, el pago de intereses cuales pueden ser a mediano y largo plazo, pero también pueden ser a un plazo indefinido.

La característica de los instrumentos de renta fija es que la rentabilidad se conoce de antemano, puesto que la tasa de interés es una tasa fija. Esto, para el comprador, se traduce en un ahorro. Todos los instrumentos de renta fija son emitidos en forma seriada, de acuerdo a ciertas características comunes de tasa de interés, plazo, fecha de emisión 0 de vencimiento y cortes, entre otros (Bolsa de Santiago, 2011)

\section{Renta Variable:}

Los instrumentos de renta variable son instrumentos representativos de capital, que se caracterizan por tener un alto riesgo y un retorno que está ligado a las utilidades obtenidas por la empresa en la cual se invirtió y por las ganancias de capital obtenidas por la diferencia entre el precio de compra y venta (Bolsa de Santiago, 2011)

\section{ACCIÓN.}

Se entiende por acción a un instrumento de renta variable, los cuales son emitidos por sociedades anónimas y en comandita por acciones. Las acciones representan, para los accionistas, un título de propiedad sobre una parte 0 fracción del patrimonio de una empresa, es decir, aquel que compra una acción pasa a ser dueño de una parte de la empresa que emite la acción.

Las acciones, desde el punto de vista de la empresa emisora, son una alternativa de financiamiento para adquirir recursos a plazo indefinido. Desde el punto de vista de aquel que compra las acciones, es una alternativa de ahorro a corto, mediano y largo plazo o mejor dicho a plazo indefinido. Esto dependerá de las razones por las cuales el accionista compra tales acciones (Bolsa de Santiago).

\section{Métodos de Valorización de Acciones}

Las acciones corresponden a un título de propiedad, respecto del patrimonio de una empresa. Las acciones no solo representan un instrumento de ahorro e inversión, sino también son un título de propiedad, respecto del patrimonio de una empresa. Así, cada vez que 
se compra una acción, se pasa a ser propietario de una parte de la empresa, lo que se relaciona directamente con la cantidad de acciones que tenga. Por lo tanto, mientras más acciones se tengan de una compañía, mayor será el porcentaje de la propiedad que se controle. A su vez, las empresas emiten acciones como una forma de obtener financiamiento para sus distintos proyectos.

Cuando una empresa cotiza en Bolsa, sus acciones pueden negociarse en el mercado bursátil; los compradores y vendedores determinan el precio de las acciones. El resultado de multiplicar el precio de la acción en el mercado por el número de acciones existentes es igual al valor bursátil o capitalización de una empresa. Este criterio es muy útil para determinar el valor real de una empresa. La determinación del precio de las acciones de las empresas supone, en definitiva, la valoración que hace el mercado sobre las expectativas de las empresas que cotizan. En este sentido, la Bolsa puede considerarse como un "barómetro" de la economía. BCl (2011)

Básicamente, los métodos de valoración de acciones que existen, se pueden clasificar en las siguientes categorías:

\section{Métodos basados en el valor patrimonial:}

Son aquellos que determinan el valor de la compañía en función de su balance. Por tanto, se trata de una valoración estática, que únicamente tiene en cuenta la situación de la sociedad en un momento concreto y determinado. (Educación financiera en la red, 2011)

\section{Métodos basados en el descuento de flujos de caja:}

El valor de la compañía se calcula como el valor actual neto de sus flujos futuros de dinero. Así, este método incide en el potencial de la entidad para generar recursos. (Educación financiera en la red, 2011).

Métodos basados en ratios bursátiles: La ratio por excelencia es la PER (Precio por acción/Beneficio por acción), si bien hay otros, comúnmente usados, como:

\section{Rentabilidad por dividendo:}

Dividendo por acción / Precio por acción.

Precio/Valor contable: compara el valor de mercado de la compañía con su valor contable, esto es, indica en qué proporción valora el mercado el valor en libros de la sociedad. (Educación financiera en la red, 2011)

\section{ROE (ReturnOnEquity o rentabilidad financiera):}

mide la capacidad de la sociedad para generar beneficios con los recursos propios de los accionistas (capital social + reservas)

$\mathrm{ROE}=$ Beneficios / Fondos Propios. (Educación financiera en la red, 2011)

\section{Deuda/EBITDA:}

Esta ratio incorpora una indicación sobre la capacidad de la entidad de contraer deuda adicional y de refinanciar la que vence. Es, por tanto, una ratio que mide el apalancamiento (deuda contraída). El EBITDA representa el margen 0 resultado bruto de la explotación, es decir, el beneficio antes de intereses, impuestos y amortizaciones. Esta ratio nos indica cuántos ejercicios serán necesarios para cancelar toda la deuda, utilizando el resultado de la explotación. (Educación financiera en la red, 2011)

\section{Tipo de acciones}

Existen diferentes tipos de acciones, según la Bolsa de Santiago (2011), que pueden ser transadas compradas 
0 vendidas:

\section{Acciones Ordinarias:}

Son acciones que confieren iguales derechos a los socios 0 accionistas, en cuanto a voto y recibir un dividendo con base en las utilidades actuales, previa aprobación por parte de la asamblea.

\section{Acciones Preferenciales:}

Tipo de acción que otorga el derecho a voto solo en las asambleas generales 0 extraordinarias de accionistas. Se denominan Preferenciales porque de acuerdo a la ley se les debe otorgar prelación en el pago de utilidades y del patrimonio social, en el caso de liquidación, respecto de las acciones ordinarias.

\section{Acciones a la Orden:}

Son las trasmisibles mediante la entrega del título, debidamente endosado por su titular.

\section{Acciones al Portador:}

Son las suscritas anónimamente. Pueden ser traspasadas por simple compraventa en una bolsa de valores. Otorgan a su poseedor el carácter de socio en la proporción por ellas representadas. Se transmiten por la simple entrega del título.

\section{Motivos que inducen a la compra de acciones}

Existen dos tipos de razones por las cuales se invierte en acciones, según la Bolsa de Santiago:

\section{Rentabilidad:}

Es la razón básica para invertir en la obtención de una renta por un periodo de tiempo determinado. Esta renta puede provenir de variaciones de precios 0 dividendos. La utilidad que proviene de las variaciones de precios recibe el nombre de ganancia de capital y, en la mayoría de los casos, es el componente principal de la rentabilidad obtenida en el mercado de acciones. Los dividendos también inciden en la rentabilidad, ya que estos corresponden a distribuciones periódicas que efectúan las compañías de la utilidad de un ejercicio determinado. (Bolsa de Santiago, 2011)

\section{Control:}

Es el deseo de control el que induce a la compra de acciones. Está relacionado con la posibilidad de obtener el manejo de una empresa, a través de compras de paquetes importantes de acciones en el mercado. Permite formar parte del directorio de una compañía y, por esa vía, participar de las principales decisiones de la empresa. (Bolsa de Santiago, 2011)

\section{OFERTA PÚBLICA DE DQUISICIÓN DE ACCIONES (OPA)}

La OPA, según el Ministerio de Hacienda (2000), es una oferta de compra de acciones realizada a través de una sociedad, la cual formula abiertamente su pretensión de adquirir una parte 0 la totalidad de los títulos de otra compañía que cotiza en bolsa. La oferta va dirigida a todos aquellos accionistas que posean acciones en la sociedad, a los que se le ofrece un precio determinado de adquisición.

La mayoría de las veces, cuando una sociedad formula una OPA, persigue el fin de tomar el control de otra sociedad o para adquirir la totalidad de las acciones 0 convertirse en propietario de un importante paquete que le abra las puertas del control de la empresa.

\section{Tipos de OPA}

Existen dos tipos de ofertas públicas de adquisición de acciones: las amistosas y las hostiles. Cuando existe conocimiento anterior y aprobación 0 acuerdo de la cúpula directiva de la sociedad afectada es llamada 
amistosa y, aquella que se realiza sin conocimiento previo o aceptación de la sociedad afectada y que a veces puede generar el lanzamiento de una contraOPA, por parte de la sociedad o de un caballero blanco, es llamada hostil. (Muñiz, 2010).

También existe la contra OPA, que es la oferta que lanza una sociedad para la adquisición de una empresa sobre la que ya pesa una OPA, cuyo plazo de aceptación aún no ha finalizado. El objetivo de la contra OPA, normalmente presentada por una sociedad afín, (denominada «caballero blanco») a la empresa afectada, es evitar que la compañía que lanzó la primera oferta se haga con la mayoría de las acciones. (Muñiz, 2010)

\section{Información a revelar cuando se lanza una OPA}

Según la Ley 19.705, el mínimo de información que deberá contener el prospecto es:

a) Individualización de los ofertantes, ya sean personas naturales o jurídicas. Si son las segundas, deben señalar:

- $\quad$ El nombre, cargo y domicilio, de sus directores, gerentes, ejecutivos principales y administradores.

- Participación en otras sociedades e individualización de las personas relacionadas del oferente.

- $\quad$ Descripción financiera, jurídica y de los negocios del oferente 0 de sus controladores.

El oferente deberá fijar un domicilio en el territorio nacional.

b) Debe incluir el número de valores a los que se extiende la oferta, es decir, las acciones que pretende adquirir la sociedad compradora. También debe incluirse el mínimo necesario, para que la oferta tenga éxito.

c) Forma de pago y condiciones, es decir, cómo se pagarán las acciones que se adquieran mediante la OPA. Estas contraprestaciones pueden hacerse en dinero 0 mediante valores del oferente (admitidos a cotización). Se deben indicar en forma precisa.

d) Vigencia de la oferta y procedimiento para aceptarla. Se indicarán con precisión aquellos antecedentes 0 documentos que deberán acompañar los accionistas interesados, en el momento de entregar sus acciones.

e) Forma y oportunidades en que los oferentes adquirieron las acciones que poseen al inicio de la oferta, si fuere el caso; y relaciones existentes con otros controladores de la sociedad 0 accionistas mayoritarios, en su caso.

f) Forma de financiar el pago del precio de las acciones que sean adquiridas al final de la oferta.

g) Garantía constituida por los oferentes e individualización del encargado de su custodia, formalización, ejecución, monto y forma de garantía. 
h) Condiciones 0 eventos que puedan provocar la retractación de la oferta.

i) Individualización completa y domicilio del tercero que el oferente hubiere designado para que organice 0 administre la oferta, debiendo precisarse las facultades que se le hayan otorgado.

j) Individualización completa y domicilio de los asesores del oferente, personas 0 profesionales independientes.

k) Otras claúsulas que disponga la superintendencia de valores y seguros, mediante normas generales.

\section{METODOLOGÍA.}

La investigación es de tipo cuantitativo, con alcance correlacional, basado en la información disponible relacionada con las Ofertas Públicas de Adquisición de acciones del sector bancario.

Para el estudio, se recogen antecedentes sobre las ofertas públicas de adquisición de acciones, de cuatro bancos chilenos (BBVA, Banco Chile, Corpbanca y Grupo Security S.A.), que han participado en el mercado bursátil desde el año 2004, debido a que entre estos años los bancos comienzan a realizar ofertas públicas. La muestra que se obtendrá será la misma cantidad de transacciones en vigencia de la OPA, antes y después de la publicación de ella.

Del total de las OPA registradas, la muestra incluye, solamente, las empresas objetivo que cotizan en bolsa y que cumplan los requisitos que se mencionan:

- $\quad$ Que no sean OPA de exclusión.
- $\quad$ Empresas que dispongan de suficientes datos, que permitan efectuar las estimaciones necesarias y analizar el efecto de la OPA.

- $\quad$ No puede verse implicada en otra OPA, ni como empresa objetivo, ni como oferente.

Luego de obtener la información, se podrá analizar mediante gráficos de dispersión la fluctuación de las acciones y su comportamiento. Posteriormente, se realizará una prueba no paramétrica "Kolmogorov-Smirnov", que proporcionará los resultados de normalidad, para saber qué tipo de coeficiente de correlación se debe aplicar a la muestra en estudio. Las dos alternativas son: para una muestra con comportamiento normal Pearson y para una muestra con comportamiento no normal se aplicará Kendall.

\section{RESULTADOS}

\section{Aplicación y Análisis prueba de Kolmogorov-Smirnov}

Se realiza el análisis de la prueba de Kolmogorov- Smirnov, la cual se efectúa en base a una distribución teórica correspondiente a la normal. El propósito de la prueba es verificar la hipótesis de distribución normal para los bancos, con el objeto de saber qué prueba de correlación se ajusta y se debe aplicar a la muestra en estudio, para que los resultados que se obtengan de la aplicación sean fiables.

Para el análisis de la prueba de Kolmogorov- Smirnov, es importante aclarar que las hipótesis son planteadas sólo para dar validez a la teoría de distribución normal para los bancos y no para la investigación. Para esto se utiliza un grado de significancia del 0,05. Las hipótesis planteadas para la prueba son las siguientes: 
Ho: $P$ valor $>\alpha$ : Normal, Prueba de Correlación Pearson.

H1: P valor < $\alpha$ : No Normal, Prueba de correlación W Kendall

\section{Dónde:}

$\mathbf{P}=$ Significación Asintótica Bilateral

$\boldsymbol{\alpha}=$ Grados de Significancia del 0,05

Cuadro N 2 Prueba de Kolmogorov-Smirnov para una muestra del Banco Chile

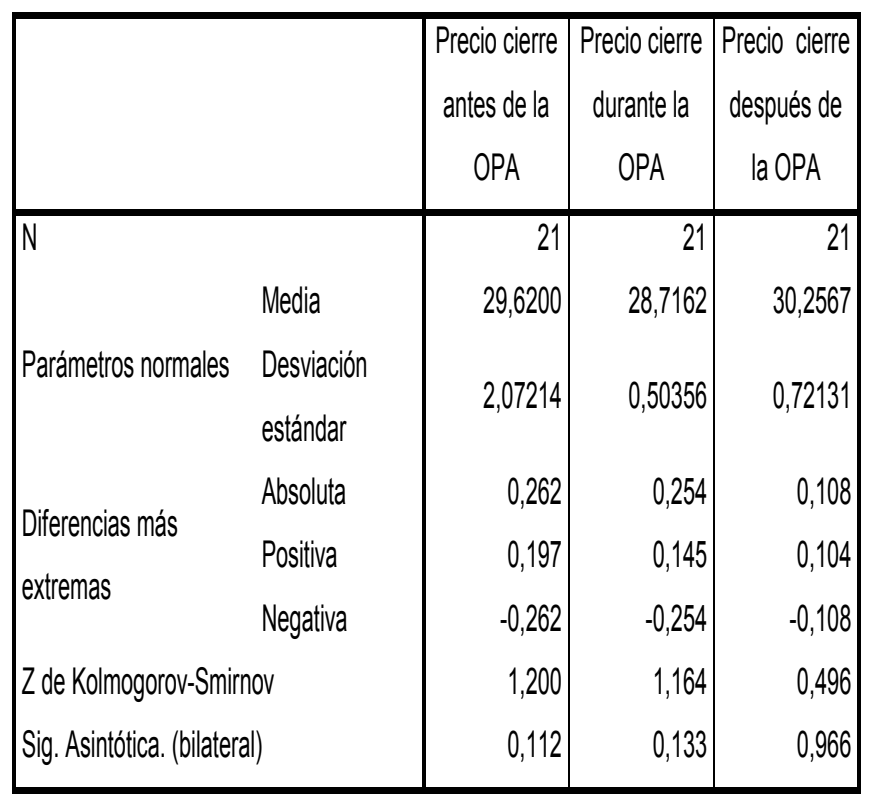

Fuente : Propia en base a información proporcionada por Consorcio Corredores de Bolsa (2012) 
Cuadro No 3 Prueba de Kolmogorov-Smirnov para una muestra del Banco

Security

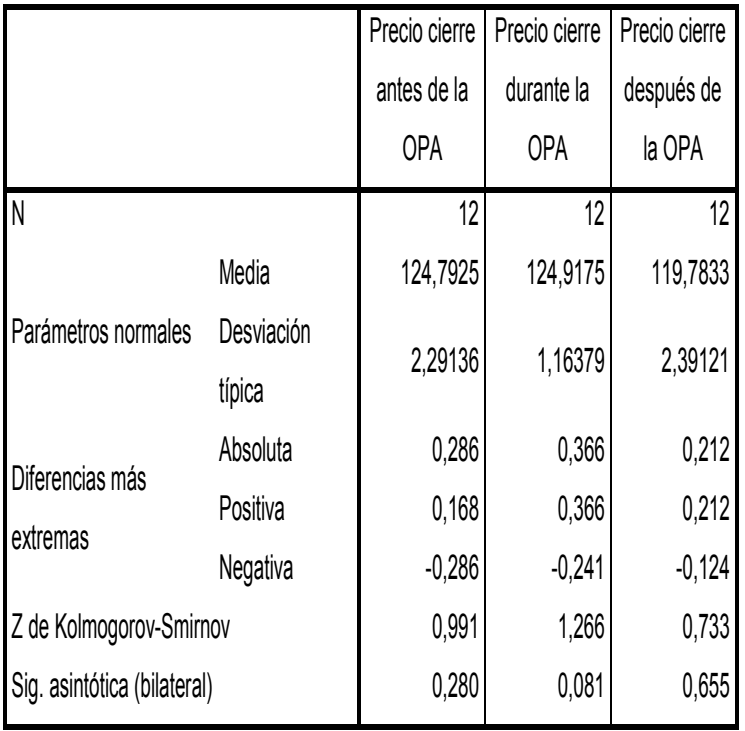

Fuente : Propia en base a información proporcionada por Consorcio Corredores de Bolsa (2012)

Cuadro $\mathrm{N}^{\circ} 4$ Prueba de Kolmogorov-Smirnov para una muestra del Banco BBVA

\begin{tabular}{|c|c|c|c|c|}
\hline & & $\begin{array}{l}\text { Precio cierre } \\
\text { antes de la } \\
\text { OPA }\end{array}$ & $\begin{array}{c}\text { Precio cierre } \\
\text { durante la } \\
\text { OPA }\end{array}$ & $\begin{array}{c}\text { Precio cierre } \\
\text { después de } \\
\text { la OPA }\end{array}$ \\
\hline $\mathrm{N}$ & & 10 & 10 & 10 \\
\hline & Media & 1522,5 & 1540,6 & 1503,0 \\
\hline Parámetros normales & $\begin{array}{l}\text { Desviación } \\
\text { típica }\end{array}$ & 32,93512 & 3,53396 & 52,50397 \\
\hline ijc mác & Absoluta & 0,302 & 0,367 & 0,277 \\
\hline & Positiva & 0,202 & 0,367 & 0,185 \\
\hline extilinas & Negativa & $-0,302$ & $-0,231$ & $-0,277$ \\
\hline Z de Kolmogorov-Smi & & 0,956 & 1,162 & 0,877 \\
\hline Sig. asintótica (bilater & & 0,320 & 0,134 & 0,426 \\
\hline
\end{tabular}

Fuente : Propia en base a información proporcionada por Consorcio Corredores de Bolsa (2012) 
Cuadro $N^{0} 5$ Prueba de Kolmogorov-Smirnov para una muestra del Banco

\section{Corpbanca}

\begin{tabular}{|ll|r|r|r|}
\hline & $\begin{array}{r}\text { Precio cierre } \\
\text { antes de la } \\
\text { OPA }\end{array}$ & $\begin{array}{c}\text { Precio cierre } \\
\text { durante la } \\
\text { OPA }\end{array}$ & $\begin{array}{r}\text { Precio cierre } \\
\text { después de } \\
\text { la OPA }\end{array}$ \\
\hline $\mathrm{N}$ & Media & 3,0364 & 3,0018 & 2,9991 \\
Parámetros normales & Desviación & 0,01804 & 0,01779 & 0,02023 \\
& típica & 0,183 & 0,268 & 0,209 \\
Diferencias más & Absoluta & 0,183 & 0,268 & 0,209 \\
extremas & Positiva & $-0,180$ & $-0,125$ & $-0,173$ \\
Z de Kolmogorov-Smirnov & 0,608 & 0,889 & 0,694 \\
Sig. asintótica (bilateral) & 0,854 & 0,408 & 0,721 \\
\hline
\end{tabular}

Fuente : Propia en base a información proporcionada por Consorcio Corredores de Bolsa (2012)

Según los resultados obtenidos en el muestreo realizado al Banco de Chile, Banco Security, Banco BBVA, Banco Corpbanca antes, durante y después de la OPA, el valor del nivel crítico (Sig. Asintótica bilateral) es mayor a los grados de significancia $(0,05)$, por lo que se acepta la hipótesis de normalidad y se concluye que las muestras se ajustan a una distribución normal, donde la prueba más acorde a cada distribución es la de Pearson.

A continuación se observan dos cuadros de resumen que muestran los resultados obtenidos, luego de aplicar la prueba estadística de Correlación Pearson a los cuatro bancos objeto de estudio, en función de la variación de las acciones, según su precio de cierre. 


\section{Cuadro $N^{\circ} 6$ Resumen Correlación Pearson}

\begin{tabular}{|c|c|c|c|c|}
\hline $\begin{array}{l}\text { Bancos } \\
\text { Periodos }\end{array}$ & $\begin{array}{l}\text { Banco } \\
\text { Chile }\end{array}$ & $\begin{array}{l}\text { Banco } \\
\text { Security }\end{array}$ & Banco BBVA & $\begin{array}{c}\text { Banco Corp- } \\
\text { banca }\end{array}$ \\
\hline $\begin{array}{c}\text { Correlación } \\
\text { Antes } \\
\text { - } \\
\text { Durante }\end{array}$ & $\begin{array}{c}\text { Coef. } \\
\text { Correlación } \\
-0,432 \\
\text { No existe } \\
\text { Correlación } \\
\text { No significativa }\end{array}$ & $\begin{array}{c}\text { Coef. } \\
\text { Correlación } \\
-0,333 \\
\text { No existe } \\
\text { Correlación } \\
\text { No significativa }\end{array}$ & $\begin{array}{c}\text { Coef. } \\
\text { Correlación } \\
0,186 \\
\text { No existe } \\
\text { Correlación } \\
\text { No significativa }\end{array}$ & $\begin{array}{c}\text { Coef. } \\
\text { Correlación } \\
-0,414 \\
\text { No existe } \\
\text { Correlación } \\
\text { No significativa }\end{array}$ \\
\hline $\begin{array}{c}\text { Correlación } \\
\text { Durante } \\
\text { - } \\
\text { Después }\end{array}$ & $\begin{array}{c}\text { Coef. } \\
\text { Correlación } \\
0,765 \\
\text { Existe } \\
\text { Correlación } \\
\text { No significativa }\end{array}$ & $\begin{array}{c}\text { Coef. } \\
\text { Correlación } \\
0,595 \\
\text { Existe } \\
\text { Correlación } \\
\text { Significativa }\end{array}$ & $\begin{array}{c}\text { Coef. } \\
\text { Correlación } \\
0,169 \\
\text { No existe } \\
\text { Correlación } \\
\text { No significativa }\end{array}$ & $\begin{array}{c}\text { Coef. } \\
\text { Correlación } \\
-0,745 \\
\text { Existe } \\
\text { Correlación Inversa } \\
\text { Significativa }\end{array}$ \\
\hline $\begin{array}{c}\text { Correlación } \\
\text { Antes } \\
\text { - } \\
\text { Después }\end{array}$ & $\begin{array}{c}\text { Coef. } \\
\text { Correlación } \\
-0,810 \\
\text { Existe } \\
\text { Correlación Inversa } \\
\text { Significativa }\end{array}$ & $\begin{array}{c}\text { Coef. } \\
\text { Correlación } \\
-0,420 \\
\text { No existe } \\
\text { Correlación } \\
\text { No significativa }\end{array}$ & $\begin{array}{c}\text { Coef. } \\
\text { Correlación } \\
0,609 \\
\text { Existe } \\
\text { Correlación } \\
\text { No significativa }\end{array}$ & $\begin{array}{c}\text { Coef. } \\
\text { Correlación } \\
0,291 \\
\text { No existe } \\
\text { Correlación } \\
\text { No significativa }\end{array}$ \\
\hline
\end{tabular}

Fuente: Propia elaborado en base a resultados obtenidos de la investigación

El cuadro muestra el índice de correlación arrojado por la prueba de correlación de Pearson de cada periodo, también se muestra, según los parámetros planteados en la investigación, si la correlación es existente 0 no.

\section{Cuadro $N^{\circ} 7$ Resumen Coeficiente de Variación Porcentual}

\begin{tabular}{|c|c|c|c|c|}
\hline $\begin{array}{l}\text { Bancos } \\
\text { Periodos }\end{array}$ & $\begin{array}{l}\text { Banco } \\
\text { Chile }\end{array}$ & $\begin{array}{l}\text { Banco } \\
\text { Security }\end{array}$ & Banco BBVA & $\begin{array}{c}\text { Banco Corp- } \\
\text { banca }\end{array}$ \\
\hline $\begin{array}{c}\text { Variación } \\
\text { Antes } \\
\text { - } \\
\text { Durante }\end{array}$ & $\begin{array}{c}\text { Coef. Variación } \\
-3,05 \% \\
\text { Baja el Valor } \\
\text { No significativa }\end{array}$ & $\begin{array}{c}\text { Coef. Variación } \\
0,1 \% \\
\text { Sube el Valor } \\
\text { No significativa }\end{array}$ & $\begin{array}{c}\text { Coef. Variación } \\
1,2 \% \\
\text { Sube el Valor } \\
\text { No significativa }\end{array}$ & $\begin{array}{c}\text { Coef. Variación } \\
-0,01 \% \\
\text { Baja el Valor } \\
\text { No significativa }\end{array}$ \\
\hline $\begin{array}{c}\text { Variación } \\
\text { Durante } \\
\text { - } \\
\text { Después }\end{array}$ & $\begin{array}{c}\text { Coef. Variación } \\
5,36 \% \\
\text { Sube el Valor Signi- } \\
\text { ficativa }\end{array}$ & $\begin{array}{c}\text { Coef. Variación } \\
-4,1 \% \\
\text { Baja el Valor } \\
\text { No significativa }\end{array}$ & $\begin{array}{c}\text { Coef. Variación } \\
-2,44 \% \\
\text { Baja el Valor } \\
\text { No significativa }\end{array}$ & $\begin{array}{c}\text { Coef. Variación } \\
-0,08 \% \\
\text { Baja el Valor } \\
\text { No significativa }\end{array}$ \\
\hline $\begin{array}{c}\text { Variación } \\
\text { Antes } \\
\text { - } \\
\text { Después }\end{array}$ & $\begin{array}{c}\text { Coef. Variación } \\
2,14 \% \\
\text { Sube el Valor } \\
\text { No significativa }\end{array}$ & $\begin{array}{c}\text { Coef. Variación } \\
-4,0 \% \\
\text { Baja el Valor } \\
\text { No significativa }\end{array}$ & $\begin{array}{c}\text { Coef. Variación } \\
-1,28 \% \\
\text { Baja el Valor } \\
\text { No significativa }\end{array}$ & $\begin{array}{c}\text { Coef. Variación } \\
-1,2 \% \\
\text { Baja el Valor } \\
\text { No significativa }\end{array}$ \\
\hline
\end{tabular}

Fuente: Propia elaborado en base a resultados obtenidos de la investigación

El cuadro toma en consideración el coeficiente de variación porcentual, el cual indica la variación experimentada en base a la media de los distintos períodos. Además, en función del precio de cierre, indica si sube o baja el valor de la acción y si la variación es significativa o no. 


\section{DISCUSIÓN DE RESULTADOS.}

Antes de comenzar la discusión de los resultados, se debe esclarecer algunos aspectos, respecto al propósito de este artículo, el primero de ellos es comprobar la siguiente hipótesis:

"La publicación de Oferta Pública de Adquisición de Acciones (OPA), produce efectos significativos en la variación del valor de las acciones de las empresas objetivo".

El otro es considerar ciertos planteamientos establecidos dentro del marco teórico, y que tienen relación con el coeficiente de correlación de Pearson, el cual indica la correlación existente entre dos variables a través de un valor numérico. Sobre este valor numérico, se puede establecer si entre las variables existe correlación o no, o si esta correlación es perfecta o nula. Para establecer esta medida se definieron los siguientes parámetros:

Cuadro $N^{\circ} 8$ Parámetros Coeficiente de Correlación Pearson.

\begin{tabular}{|c|c|}
\hline Correlación & Coef. Numérico \\
\hline Existe & Mayor a 0,5 \\
\hline No existe & Menor a 0,5 \\
\hline Perfecta & Igual a 1 \\
\hline Nula & Igual a 0 \\
\hline
\end{tabular}

\section{Fuente: Propia elaborado en base a resultados obtenidos de la investigación}

Considerando estos dos aspectos, se puede dilucidar que para cumplir con la hipótesis establecida, es necesario comprobar que entre los periodos "antes - durante" y "durante - después" no existe correlación, es decir, es menor a 0,5 . Por el contrario, si la medida de correlación es mayor a 0,5 resulta que si existe correlación, esto indicaría que el comportamiento de las variables es similar y que no existe ningún tipo de efecto sobre las acciones, debido a la OPA.

Otro punto interesante a considerar tiene relación con la "Significación", la cual es entregada para esta investigación por el coeficiente de variación calculado sobre la media de cada período en estudio y que debe ser comparada con los grados de significancia. Para este estudio, el valor a considerar fue de $5 \%$ de variación en el precio de la acción.

Por último, y lo más importante es indicar que para este estudio fue necesario establecer un parámetro de comportamiento "normal", el cual fue llamado "antes". Esto fue necesario para comparar si la variación existente en los períodos, durante y posterior a la vigencia de la OPA, fueron diferentes.

A continuación se explica, mediante la evidencia obtenida, las variaciones experimentadas en las acciones cotizadas en el mercado bursátil que se han generado, producto del lanzamiento de una OPA, éstas se explicarán, separadamente, por casos.

\section{Primer Caso; comportamiento "antes} - durante".

Al observar el cuadro de Correlación Pearson y el cuadro de Coeficiente de Variación Porcentual, se puede apreciar que la correlación que existe entre los bancos; Security, Banco de Chile, BBVA y Corpbanca, con respecto al comportamiento del período denominado como "antes - durante", corresponde a una correlación cuyos índices son menores a 0,5, esto quiere decir, que si se toman como referencia el comportamiento del período "antes" de la OPA, como un comportamiento normal, las acciones en su conjunto indican que no existe correlación, 0 
sea, se cumple lo que se busca desde los inicios de la investigación, que es que el período durante la vigencia se comporte distintamente a lo que ocurría normalmente. Ahora bien, efectivamente se puede afirmar que sí existe una diferencia entre el comportamiento del valor de las acciones en la bolsa de estos dos períodos (antes-durante), pero ésta no es significativa, según el Coeficiente de variación, el cual indica un porcentaje para los Bancos menor al $5 \%$.

Descrito lo anterior, la evidencia recogida después de analizar cada banco indica que la hipótesis planteada no se cumple. Aún así se puede observar que la OPA influye en la variación de las acciones durante el periodo de vigencia de ésta, pero no significativamente, ni tampoco de forma idéntica para todos los bancos, ya que el precio de las acciones en algunos casos sube y en otros baja.

\section{Segundo Caso; comportamiento "durante-después"}

Lo que se busca en este caso es que exista correlación entre lo que sucedía durante la vigencia de la OPA y después de ésta. Esto indicaría que las acciones se siguen comportando de igual forma que durante la vigencia, pero de forma distinta a lo que se considera normal.

Si se observa el cuadro Correlación Pearson y Coeficiente de Variación Porcentual, se compara el coeficiente de correlación de los cuatro bancos, considerando los períodos "durante después", se observa que las acciones se comportan de manera distinta en todos los bancos, por lo tanto, no se puede afirmar que después de la vigencia de la OPA, experimenten algún tipo de variación distinta a lo que se consideró como un comportamiento normal, es decir, en este caso la hipótesis no se cumple.

También se puede observar, a través del coeficiente de variación porcentual, que después de terminada la vigencia de la OPA, las acciones del Banco Chile son las únicas que suben su precio significativamente. En cambio, en los otros tres bancos, las acciones bajan su valor, pero esta baja no es significativa.

Respecto a lo anterior, en los Bancos de Chile y Security, se puede observar que existe correlación entre lo que sucede en los períodos "durante" y "después", esto quiere decir que las acciones de cada banco siguen comportándose igual a como se comportaban durante la vigencia de la OPA, pero en su conjunto, se comportan distinto al período "antes" de la OPA, lo cual es considerado el comportamiento normal.

En el Banco BBVA, según el coeficiente de correlación observado se puede ver que no existe correlación entre lo que sucede en el período "durante" la oferta, esto quiere decir que las acciones no se siguen comportando de igual forma.

El Banco Corpbanca, al contrario de lo que sucede en los otros tres bancos, entrega un coeficiente de correlación negativo, el cual indica que las acciones se comportan con una correlación inversa.

Finalmente, después de obtener la evidencia, se puede afirmar que la hipótesis no se cumple para el período analizado "durante y después" y que el comportamiento de las acciones después del término de la vigencia de la OPA es desigual en los diferentes bancos. En conclusión, en el período "después" no existe un efecto en la variación de las acciones, producto de la OPA. 
Tercer caso, comportamiento "antesdespués"

Por último, se observa la relación que existe entre el período "antes" y "después" de la oferta. Los Bancos Chile y Security siguen el mismo comportamiento del período denominado "durante" la OPA, esto se puede concluir a través del índice de correlación negativo, el cual indica que la tendencia de las acciones va en diferente dirección.

En el Banco BBVA se obtiene un Coeficiente de correlación alto y positivo, lo que quiere decir que las acciones se comportan de forma igual en el período "antes" y en el período "después".

Respecto al Banco Corpbanca, se puede concluir que las acciones se comportan de forma distinta en estos dos períodos.

Si se observa el coeficiente de variación porcentual, éste muestra que solo en el Banco de Chile suben las acciones con respecto al período considerado como normal, en cambio en los otros bancos el valor de las acciones baja, con respecto al período anterior a la vigencia de la OPA.

Entonces, se puede afirmar que mediante la evidencia recabada, la hipótesis planteada para la investigación se rechaza, puesto que las acciones tienen un comportamiento anormal durante la vigencia de la OPA, pero este comportamiento no es significativo. Por tanto, el lanzamiento de una OPA sí tiene efectos en la variación de las acciones cotizadas en bolsa, para el sector bancario, pero ésta no es significativa, ni tampoco se puede asegurar que tenga un solo efecto, 0 sea, no se puede afirmar que el lanzamiento de una OPA tiene como efecto que las acciones solo suban o solo bajen de valor. 


\section{CONCLUSIÓN.}

Las OPA resultan particularmente atractivas, fundamentalmente por dos motivos. En primer lugar, porque es una operación importante y poco habitual en la vida de una empresa y, en segundo lugar, porque el anuncio de este tipo de operaciones tiene un efecto importante en el precio de las acciones de la empresa objetivo.

Los objetivos iniciales, que dieron origen a la idea de normar las ofertas públicas de adquisición de acciones, se han cumplido en forma amplia. Los prospectos exigidos han permitido entregar la información necesaria para esta investigación sobre las características de la OPA. La identificación del oferente, sus planes de negocios para la sociedad y los plazos de duración. Esto también ha permitido que las OPA representen un mecanismo más transparente y competitivo, para adquirir el control de una sociedad que hace oferta pública de sus acciones.

Los datos utilizados en esta investigación, corresponden a los precios de cierre histórico del sector bancario, que realizan oferta pública. Se analizaron los precios de cierre de las acciones respectivas, de aproximadamente un mes antes y un mes después del lanzamiento de la OPA, considerando la cantidad de transacciones generadas durante ésta.

Se realizó el análisis de la prueba de Kolmogorov- Smirnov, la cual se efectuó en base a una distribución teórica correspondiente a la normal. El propósito de la prueba fue verificar la hipótesis de distribución normal para los cuatro bancos en estudio, con el objeto de saber qué prueba de correlación se ajusta, la cual dio como resultado que los cuatro bancos en estudio pertenecian a una distribución normal y para todos estos se aplicó la prueba de correlación Pearson.

El problema que se presenta es que para analizar un efecto particular se debe verificar que no exista otro evento relevante durante días cercanos, antes o después, del lanzamiento de la OPA, tal como un hecho económico que podría sesgar los resultados afectando los precios accionarios y, consecuentemente, el valor de la empresa. Por esto, se revisaron los datos existentes y se descartó algún tipo de factor externo que pudiera afectar la investigación.

El principal aporte de este estudio, es el haber trabajado con datos de transacciones diarias, lo permitió, por una parte, detectar el momento en el que se producen las variaciones, como consecuencia de la publicación de la OPA y, por otra parte, localizar 
posibles efectos en el comportamiento de las acciones en los períodos antes, durante y después.

Por último, en relación a los aspectos observables del mercado bursátil y a lo que los estudios muestran, a través de las pruebas realizadas, se pudo concluir a través de su análisis, que el lanzamiento de una OPA influye en el comportamiento de las acciones en el período que comprende la duración de la oferta. Entonces, se puede afirmar que la publicación de la noticia (de inicio de la OPA) provoca, como era de esperar, variaciones anormales en las empresas objetivo, concentrada casi en su totalidad durante el período de vigencia de ésta, donde no se encuentra correlación.

No obstante, el efecto que la noticia tiene sobre las distintas empresas es diferente, incluso en algunas de ellas la divulgación de la información produce incrementos en el precio de la acción y en otras una baja, lo que indicaría mayores asimetrías una vez conocida la información.

Al utilizar el coeficiente de variación aplicada sobre la media de los valores de las acciones de cada banco en estudio, también se pudo concluir que las variaciones no son significativas, estadísticamente, es decir, tampoco hubo un efecto importante en el caso de los precios de estas acciones.

Finalmente, la hipótesis planteada y objeto de la investigación, que afirmaba que: "La publicación de Oferta Pública de Adquisición de Acciones (OPA) produce efectos significativos en la variación del valor de las acciones de las empresas objetivo". Luego del estudio realizado, se pone de manifiesto que no es válida y solo se pude afirmar que existe una variación anormal en el mercado bursátil, que debe ser considerada, tanto por parte de los inversores, como por parte del regulador del mercado ante la realización de una OPA, pero esta variación anormal no es significativa.

Los análisis efectuados han permitido conocer mejor el comportamiento del mercado y la formación de precios ante un acontecimiento tan importante como es una OPA, permitiendo afirmar que dado el comportamiento de las acciones, la publicación de la noticia tiene efectos importantes en la mayoría de los aspectos bursátiles de las empresas objetivo de la misma. 


\section{BIBLIOGRAFÍA.}

- $\quad$ BCl. (2011)¿Qué son las acciones? Santiago de Chile: Autor. Consultado en 29/12/11, De http://www. bci.cl/inversiones/acciones/queacciones_ act.html

- Bolsa de Santiago. (2011). Reseña Histórica. Santiago deChile: Autor. Consultado el 05/11/2011, De http://www. bolsadesantiago.com/Lists/Bolsa $\% 20$ de\%20Comercio/dispbolsacomercio. aspx?ID $=28$

- $\quad$ Corvalán, E y Muñoz, F. (2004). Atentados y su impacto en el mercado de capitales chileno. Universidad de Chile. Seminario para optar al título de Ingeniero Comercial. [PDF] CHILE, Disponible en versión electrónica en: http://www. cybertesis.cl/tesis/uchile/2004/corvalan_e/ sources/corvalan_e.pdf

- Lacén, V y Sepúlveda, A. (2004).Desarrollo de las tomas de control corporativo en Chile después de la ley de OPAs. [PDF] .CHILE: Disponible en versión electrónica en: http://www.svs.cl/sitio/ publicaciones/doc/informe_serie_doc_ trab4.pdf

- $\quad$ Ley 19.705, Regula las Ofertas Publicas de Adquisicion de Acciones (opas) y establece Regimen de Gobiernos Corporativoshttp://www.leychile.cl/ Navegar?idNorma $=179295$

- Levin, R. \& Rubin, D.(1996). Estadística para administradores. Chile: Pearson Educación de Chile LTDA.

- Ministerio de Hacienda. (2000). Ley 19.705 que regula las ofertas públicas de adquisición de acciones (OPAS) y establece régimen de gobiernos corporativos. Santiago: Ediciones Publiley.

- Ministerio de Hacienda. (2011)¿Qué es el mercado de capitales chileno?.Santiago de Chile: autor. Consultado el 10/11/11, de http://www. minhda.cl/mercado-de-capitales/preguntasfrecuentes/que-es-el-mercado-decapitales-chileno.html

- $\quad$ Muñoz, G. (2010). ¿Qué significa una OPA? .Recuperado 0ctubre 10, 2011 de la base de datos Decroly, Dehttp://decroly. com/decrolydigital/2010/05/11/\%C2\%BFq ue-significa-opa/

- $\quad$ Real Academia Española. (2009). Real Academia Española de la Lengua. $22^{\circ}$ Edición.Disponible en: http://www.rae.es/ rae.html

- $\quad$ Salinas, P. (2006). Valorización de empresas a través del método de comparables: Evidencia Chile. Seminario de título Ingeniero Comercial, mención administración. Santiago de Chile.

- Superintendencia de Valores y Seguros. (2011). Estructura de mercado. Santiago de Chile: Autor. Consultado el día 13/11/1. De, http://www.svs.cl/sitio/ mercados/valores estruclnter.php

- Valderrama, M. (2010). Control como causal de una ofertaPública de adquisición de acciones obligatoria y su regulación en Chile. Chile: Disponible en versión electrónica en:http://www. revistas.uchile.cl/index.php/RDH/article/ viewFile/17044/17766

- Vargas, A. (1995). Estadística descriptiva e inferencia. Edición II. España.Servicio de publicación Universidad de Castilla. pág. 193-199. Disponible en versión electrónica en: http://books.google.cl/books?id=RbaCwP WqjsC\&pg $=P A 193 \& l p g=P A 193 \& d q=q u$ ien + creo +el+coeficiente + de + correlac ion + de + pearson + que + a $\%$ C3\%B10\&so urce $=$ bl\&ots $=$ W02sKG7rZ\&sig $=732 \mathrm{LI}$ 48Z8YFXKUzoy6ltEYFJzQ\&hl $=e s \& \bar{s} a=X \&$ ei $=$ WyYKT4ilJqT10gH0v_y6Ag\&ved $=0 \mathrm{C}$ FEQ6AEwBg\#v= onepage \&q=quien $\% 20$ creo $\% 20$ el\% 20 coeficiente $\% 20$ de $\% 20$ correlacion\%20de\%20pearson\%20que\%20 $\mathrm{a} \% \mathrm{C} 3 \% \mathrm{~B} 10 \& \mathrm{f}=$ false 